\title{
NOTAS TAXONÓMICAS SOBRE HEMIPOGON SUBGEN. ASTEPHANOPSIS, Y DESCRIPCIÓN DE UN NUEVO GÉNERO (APOCYNACEAE, ASCLEPIADOIDEAE, ASCLEPIADEAE, METASTELMATINAE)
}

\author{
JORGE FONTELLA PEREIRA ${ }^{1}$, RENATA GABRIELLE PINHEIRO SANTOS ${ }^{2}$, MONIQUE BRITTO DE GOES ${ }^{3} \&$ \\ SERGIO ARIEL CÁCERES MORAL ${ }^{4}$
}

\begin{abstract}
Summary: Fontella Pereira, J., R. G. P. Santos, M. B. de Goes \& S. A. Cáceres Moral. 2014. Taxonomic notes on Hemipogon subgen. Astephanopsis, and description of a new genus (Apocynaceae, Asclepiadoideae, Asclepiadeae, Metastelmatinae). Bonplandia 23(1): 25-31.

Morilloa (Apocynaceae, Asclepiadoideae, Metastelmatinae) is described as a new genus close to Hemipogon sensu Decaisne and Fournier from which it is distinguished by significant morphological and biomolecular characters. Comprising four species: M. carassensis, M. furlanii, $M$. lutea and M. piranii, this new genus is restricted to Brazil, occurring in campo rupestre, cerrado and in forest borders in the States of Goiás, Bahia, Minas Gerais and São Paulo. Comments on Hemipogon subgen. Astephanopsis Malme are also provided.
\end{abstract}

Key words: Asclepiadoideae, Astephanopsis, Morilloa, new genus, taxonomy.

Resumen: Fontella Pereira, J., R. G. P. Santos, M. B. de Goes \& S. A. Cáceres Moral. 2014. Notas taxonómicas sobre Hemipogon subgen. Astephanopsis, y descripción de un nuevo género (Apocynaceae, Asclepiadoideae, Asclepiadeae, Metastelmatinae). Bonplandia 23(1): 25-31.

Morilloa (Apocynaceae, Asclepiadoideae, Metastelmatinae) se describe como un nuevo género afín a Hemipogon sensu Decaisne y Fournier, del que se separa por caracteres morfológicos y biomoleculares significativos. Comprende cuatro especies: $M$. carassensis, $M$. furlanii, M. lutea y M. piranii, restringidas a Brasil donde crecen en campos rupestres, cerrados y borde de bosques, de los estados de Goiás, Bahia, Minas Gerais y São Paulo. Se realizan consideraciones sobre Hemipogon subgen. Astephanopsis Malme.

Palabras clave: Asclepiadoideae, Astephanopsis, Morilloa, nuevo género, taxonomía.

${ }^{1}$ Departamento de Botânica, Setor de Fanerógamas, Museu Nacional-UFRJ, Bolsista do CNPq; e-mail: jofope@, mn.ufrj.br

${ }^{2}$ Departamento de Botânica, Setor de Fanerógamas, Museu Nacional-UFRJ, Colaboradora; e-mail: natagabrielle@ mn.ufrj.br

${ }^{3}$ Departamento de Botânica, Instituto de Biologia-UFRJ , Professora Substituta; e-mail: mbgoes@gmail.com

${ }^{4}$ Instituto de Botánica del Nordeste, IBONE-CONICET. Facultad de Ciencias Agrarias, Universidad Nacional del Nordeste, FCA-UNNE. C. C. 209, 3400 Corrientes, Argentina. qcaceres@gmail.com 


\section{Introducción}

Hemipogon Decne. es un género neotropical que cuenta actualmente con cerca de 18 taxones que crecen en toda América del Sur.

Decaisne (1844) lo describe por primera vez incluyendo dos especies: $H$. acerosus (typus del género) y $H$. setaceus. Desde entonces fue tratado por diversos autores (Tabla 1), destacándose: Fournier (1882, 1885), Schumann (1895, 1898), Rusby (1920), Malme (1900, 1927), Hoehne (1951), Fontella Pereira \& Marquete (1975), Fontella Pereira \& Schwarz (1983), Fontella Pereira (1987), Fontella Pereira \& Paixão (1996) y Rapini et al. (2001).

Fournier (1882) describe a Hemipogon peruvianum y posteriormente redescribe al género Hemipogon (Fournier, 1885) y a las especies de Decaisne (loc. cit.), proponiendo además los siguientes taxones: $H$. acerosus var. viridis E. Fourn., $H$. luteus E. Fourn., $H$. sprucei E. Fourn. y H. abietoides E. Fourn.

Schumann, hacia fines de ese siglo (1895), redescribe el género y en 1898 publica Hemipogon laxifolius K. Schum. una nueva especie para Brasil.

Posteriormente Malme (1900) estableció un nuevo subgénero "Astephanopsis" para el género Hemipogon incluyendo en él a las siguientes especies: Hemipogon exaltatus Malme (especie tipo de Astephanopsis, más tarde incluida en la sinonimia de $H$. sprucei), $H$. luteus, H. laxifolius y H. sprucei.

Rusby (1920) describió dos nuevas especies para la flora de Bolivia, $H$. andinum y $H$. williamsii.

Años más tarde Malme (1927) publica Astephanus carassensis una nueva especie para el estado de Minas Gerais y transfiere a $H$. luteus al género Astephanus.

Otras novedades para la flora de Brasil fueron publicadas posteriormente por Hoehne (1951) que describe a $H$. acerosus var. platyphyllus Hoehne para el estado de Mato Grosso; Fontella Pereira \& Marquete (1975) describen Astephanus grazielae Fontella \& Marquete, para el Estado de Minas Gerais; Fontella Pereira \& Schwarz (1983) incluyen Astephanus laxifolius K. Schum. en la sinonimia de Astephanus luteus (E. Fourn.) Malme.
Pocos años después Fontella Pereira (1987), siguiendo el concepto de Malme (1927), incluye algunas especies brasileñas de Hemipogon en el género Astephanus y describe tres especies nuevas: Astephanus furlanii Fontella, Astephanus semirii Fontella y Astephanus piranii Fontella. En este mismo trabajo el referido autor incluye $H$. exaltatus en la sinonimia de $A$. sprucei (actualmente $H$. sprucei; vide Tabla 1) y transfiere Astephanus gardneri E. Fourn. al género Ditassa R. Br. (Ditassa emmerichiae Fontella, nuevo nombre en virtud de la existencia de Ditassa gardneri E. Fourn.).

En otra contribución Fontella Pereira \& Paixão (1996) describen una especie nueva para el estado de Goiás, Hemipogon irwinii Fontella \& Paixão.

Éstas y otras combinaciones están resumidas en un cuadro comparativo evidenciando el estado actual de los taxones de Hemipogon y géneros afines (Tabla 1).

Otros aportes fueron realizados por Liede (1994; 1997), quien estableció una nueva circunscripción para Astephanus, excluyéndolo del neotrópico y propuso una nueva delimitación para las subtribus de Asclepiadeae, de modo que Hemipogon pasó a pertenecer a Metastelmatinae, y en consecuencia se realizaron nuevas combinaciones para adecuar las especies neotropicales a tales cambios (Rapini et al., 2001; Goyder, 2004).

Por su parte Rapini et al. (2001) en su trabajo sobre la flora de la Cadena del Espinazo transfieren, a las especies tratadas como Astephanus por Fontella Pereira (1987), a los géneros Hemipogon Decne. - H. luteus E. Fourn., H. furlanii (Fontella) Rapini, H. piranii (Fontella) Rapini - y Ditassa R. Br. - D. semirii (Fontella) Rapini y D. grazielae (Fontella \& Marquete) Rapini.

Finalmente, estudios filogenéticos recientes (Rapini et al., 2003; Liede-Schumann et al., 2005; Rapini et al. 2007; Silva et al., 2012; Liede-Schumann \& Meve, 2013), sobre la base de datos biomoleculares, revelaron que aproximadamente el $75 \%$ de las Asclepiadoideae neotropicales pertenecen a un clado formado por las subtribus Metastelmatinae, Oxypetalinae, Orthosiinae y Gonolobinae (MOOG), además de las 
Fontella Pereira \& al., Notas taxonómicas sobre Hemipogon y descripción de un nuevo género

Tabla 1. Relación de los taxones de Hemipogon y géneros afines, pasado y presente.

Taxones de Hemipogon y de otros géneros neotropicales

Identidad hasta 2013

\begin{abstract}
Astephanus carassensis Malme (1927)
Astephanus furlanii Fontella (1987)

Astephanus gardneri E. Fourn. (1885)

Astephanus grazielae Fontella \& Marquete (1975)

Astephanus laxifolius (K. Schum.) Malme (1927)

Astephanus luteus (E. Fourn.) Malme (1885)

Astephanus piranii Fontella (1987)

Astephanus semirii Fontella (1987)

Astephanus sprucei (E. Fourn.) Fontella (1987)

Hemipogon abietoides E. Fourn. (1885)
\end{abstract}

Hemipogon acerosus Decne. (1844)

Hemipogon acerosus var. platyphylla Hoehne (1951)

Hemipogon acerosus var. viridis E. Fourn. (1885)

Hemipogon andinum Rusby (1920)

Hemipogon exaltatus Malme (1900)

Hemipogon irwinii Fontella \& Paixão (1996)

Hemipogon laxifolius K. Schum. (1898)

Hemipogon luteus E. Fourn. (1885)

Hemipogon peruvianus E. Fourn. (1882)

Hemipogon setaceus Decne. (1844)

Hemipogon sprucei E. Fourn. (1885)

Hemipogon williamsii Rusby (1920)

Melinia harleyi Fontella (1994)

Metastelma hatschbachii Fontella \& Marquete (1977)

Metastelma hemipogonoides Malme (1937)
Hemipogon carassensis (Malme) Rapini (2001)

Hemipogon furlanii (Fontella) Rapini

Ditassa emmerichiae Fontella (1987)

Minaria grazielae (Fontella \& Marquete) T.U.P. Konno \& Rapini

Hemipogon luteus E. Fourn. (1885)

Hemipogon luteus E. Fourn. (1885)

Hemipogon piranii (Fontella) Rapini (2001)

Minaria semirii (Fontella) T. U. P. Konno \& Rapini (2006)

Hemipogon sprucei E. Fourn. (1885)

Hemipogon abietoides E. Fourn. (1885)

Hemipogon acerosus Decne. (1844)

Hemipogon acerosus var. platyphylla Hoehne (1951)

Hemipogon acerosus var. viridis E. Fourn. (1885)

Hemipogon peruvianus E. Fourn. (1882)

Hemipogon sprucei E. Fourn. (1885)

Hemipogon irwinii Fontella \& Paixâo (1996)

Hemipogon luteus E. Fourn. (1885)

Hemipogon luteus E. Fourn. (1885)

Hemipogon peruvianus E. Fourn. (1882)

Hemipogon setaceus Decne. (1844)

Hemipogon sprucei E. Fourn. (1885)

Hemipogon sprucei E. Fourn. (1885)

Minaria volubilis Rapini \& U. C. S. Silva (2012)

Hemipogon hatschbachii (Fontella \& Marquete) Rapini (2001)

Hemipogon hemipogonoides (Malme) Rapini (2001) recientemente reconocidas Pentacyphinae, Diplolepinae y Tassadiinae representadas, respectivamente, por Pentacyphus Schltr., Diplolepis R. Br. y Tassadia Decne. (Endress et al., 2014).

Aunque la monofilia de MOOG esté bien sustentada, las relaciones dentro de los grupos todavía no están esclarecidas. Sólo en la última década cinco subtribus fueron reconocidas: Cynanchinae K. Schum. (Liede \& Täuber, 2002), Orthosiinae Liede \& Rapini (LiedeSchumann et al., 2005), Diplolepinae Liede
\& Meve, Pentacyphinae Liede \& Meve, Tassadiinae Liede \& Meve (Endress et al., 2014), todas ellas pertenecientes anteriormente a Metastelmatinae.

De acuerdo con (Rapini et al., 2003; LiedeSchumann et al., 2005; Rapini et al., 2006; Rapini et al., 2007; Silva et al., 2012), diversos grupos de especies incluidas en Hemipogon están sin resolver y agrupadas en clados diferentes, que aunque bien sustentados molecularmente, a veces relacionan taxones morfológicamente distintos. 


\section{Materiales y Métodos}

Fueron examinados materiales provenientes de herbarios brasileños y europeos, a saber: HB, MBM, R, RB, SP, SPF, G, BM, K, M, P, TUR, W, WU, incluyendo todos los tipos de los taxones aquí estudiados (acrónimos según Thiers, 2013).

La clave dicotómica, comentarios y discusión están basados sobre el análisis de los especímenes y en las informaciones de sus etiquetas respectivas y de la literatura.

Las especies tratadas como Morilloa fueron descritas e ilustradas por Fontella Pereira (1987) y Rapini et al. (2001). De esta manera, en este trabajo, se describe sólo el género.

\section{Resultados}

\section{Morilloa Fontella, Goes \& S. Cáceres, gen. nov.}

Plantae volubiles, ramis glabris vel glabrescentibus. Folia opposita, subssessilia vel brevipetiolata, supra in basi nervi primarii 1-2 glandulis minutis instructa; laminis linearibus, linear-lanceolatis vel capillaceis, glabris vel glabrescentibus. Thyrsoideae cum cymis partialibus, extraaxillaribus, alternis, paucifloris vel multifloris, umbelfiformibus vel racemiformibus. 3-9-floribus, longipedunculatis. Flores albi vel flavi, vel viridescentes, pedicellati; calyx lobulis triangularlanceolati, adaxialiter cum glandulis in axillis; corolla urceolata vel lageniformis, inntus in parte barbata vel sericeo-puberulenta, tubo et lobis gynostegium longioribus, lobis erectis vel suberectis. Corona nulla vel vestigialis. Gynostegium sessile vel brevistipitatum; loculi antherarum secus gynostegio dispositi; retinaculum ovatum, ellipticum, obovatum vel scutiforme; caudiculae filiformes, horizontales vel obliquo-descendentibus, cum vel absque membrana hyalina; pollinia pendentia, ovata, elliptica vel oblonga, inermia y laevigata. Apendix stylare mamilata. Fructus anguste linear-lancolatus, laevigatus; semina verrucosa et comosa.

Holotypus: Morilloa lutea (E. Fourn.) Fontella, Goes \& S. Cáceres (Hemipogon luteus E. Fourn. in Mart. Fl. Bras. 6(4): 197.1885.

Genus novum Hemipogoni affinis, sed pracicpue habitu volubile, caule et ramis glabris vel glabrescentibus, foliis oppositis, cymis longe pedunculatis et fructi anguste linaer-lanceolato et laevigato differt.

El nombre de este género es un homenaje al Dr. Gilberto Morillo, del Depto. de Botánica, Facultad de Ciencias Forestales y Ambientales, Universidad de los Andes, Mérida, Venezuela, autor de numerosos e importantes trabajos sobre Asclepiadoideae (Apocynaceae).

Distribución: Brasil. Estados de Goiás, Bahia, Minas Gerais y São Paulo, donde habita en campos rupestres, cerrados, borde de bosques, en suelos pedregosos o arenosos.

\section{Clave para identificación de las especies de Morilloa}

1. Inflorescencias parciales en cimas racemiformes; lóbulos de la corola fuertemente retorcidos en espiral, más de $3 \mathrm{~mm}$ long. 2. M. furlanii

1'. Inflorescencias parciales en cimas umbeliformes; lóbulos de la corola levemente retorcidos o aparentemente valvares, hasta $2,5 \mathrm{~mm}$ long.

2. Láminas foliares lineares o linear-lanceoladas, 1-5 mm lat.; lóbulos de la corola con ápice obtuso.

3. Retináculo suboblongo o subobovado, ápice obtuso o redondeado, 0,22-0,24 mm long., 0,09-0,11 mm lat.; polinios casi el doble más largo que el retináculo, 0,32-0,38 mm long., 0,10-0,13 mm lat., caudículas desprovistas de membrana hialina.......................................................... M. carassensis

3'. Retináculo escuteliforme, ápice emarginado, 0,34-0,37 mm long., 0,17-0,20 mm lat.; polinios casi tan largos como el retináculo, o levemente más largos, 0,43-0,52 mm long, 0,13-0,17 mm lat., caudículas provistas de membrana hialina 4. M. piranii

2'. Láminas foliares filiformes o capiláceas, 0,5-1 mm lat.; lóbulos de la corola con ápice agudo 
1. Morilloa carassensis (Malme) Fontella, Goes \& S. Cáceres, comb. nov.

Astephanus carassensis Malme, Ark. Bot. 21A (12): 5. 1927; Fontella Pereira, Bradea 4(48): 381, Est. 2, Fig. J, Est. 3, Fig. L, Est. 4, Fig. U. 1987. Typus. BRASIL. Minas Gerais: Carassa, IV-1885, E. A. Vainio s.n. (holotypus TUR!).

Hemipogon carassensis (Malme) Rapini, Bol. Bot. Univ. S. Paulo 19: 140, Fig. 21 A-F. 2001. syn. nov.

\section{Morilloa furlanii (Fontella) Fontella, Goes \& S. Cáceres, comb. nov.}

Astephanus furlanii Fontella, Bradea 4(48): 381. 1987. Est. 2, Fig. G, Est. 3, Fig. N, Est. 4 Fig. S. Typus. BRASIL. Estado de Minas Gerais: Estrada de Montes Claros a Grão Mogol, a 68 km de Grão Mogol, cerrado, subarbusto $30-50 \mathrm{~cm}$ escandente, flores róseas a alvas, corola torcida, 12-IV-1981, A. Furlan et al. 686 (holotypus SPF!)

Hemipogon furlanii (Fontella) Rapini. Bol. Bot. Univ. S. Paulo 19:143, Fig. 21 Q-V. 2001. syn. nov.

\section{Morilloa lutea (E. Fourn.) Fontella, Goes \& S. Cáceres, comb. nov.}

Hemipogon luteus E. Fourn. in Mart. Fl. Bras. 6(4): 197. 1885; Rapini, Bol. Bot. Univ. São Paulo 19: 143. 2001. Typus. BRASIL. Bahia: In saxosis Serra da Lapa, I, Riedel 1341 (holotypus P!).

Hemipogon laxifolius K. Schum., Bot. Jahrb. Syst. 25 (60): 20. 1898. Typus. BRASIL. Minas Geraes: in Serra do Cipo, Schwacke 8170 (lectotypus RB!, designado por Fontella Pereira \& Schwarz, 1983). syn. nov.

Astephanus laxifolius (K. Schum.) Malme, Ark. Bot. 21 31A (12): 6. 1927. syn. nov.

Astephanus luteus (E. Fourn.) Malme, Ark. Bot. 21A (12): 6. 1927. syn. nov.

\section{Morilloa piranii (Fontella) Fontella, Goes \& S. Cáceres, comb. nov.}

Astephanus piranii Fontella, Bradea 4(48): 381. 1987. Est. 2, Fig. K, Est. 3, Fig. M, Est. 4, Fig. V. Typus. BRASIL. Minas Gerais: Município Santana do Riacho, ao longo da rodovia Belo Horizonte-
Conceição do Mato Dentro, Serra do Cipó, aprox. $18^{\circ} 55^{\prime} \mathrm{S} 43^{\circ} 54^{\prime}$ W, Santana do Pirapama, Fazenda Inhame (Serra Mineira), 20-III-1982, J. Pirani et al. CFSC-7969 (holotypus SPF!).

Hemipogon piranii (Fontella) Rapini, Bol. Bot. Univ. S. Paulo 19: 142, Fig. 21 G-J. 2001. syn. nov.

\section{Discusión y Conclusiones}

Este nuevo género es muy semejante a Hemipogon sensu Decaisne (1844) y Fournier (1885) por la corola lageniforme o urceolada y la corona ausente, pero se distingue del mismo principalmente por el tallo voluble glabro o glabrescente, las hojas opuestas, cimas parciales largamente pedunculadas y fruto estrecho (angostamente) linear-lanceolado o fusiforme, liso o estriado.

Rapini et al. (2001) en el trabajo sobre las Asclepiadoideae de la Cadena del Espinazo de Minas Gerais, presentan una clave para la identificación de $H$. carassensis, $H$. piranii, $H$. luteus y $H$. furlanii, designándolas como "especies del grupo Hemipogon luteus", lo que evidencia sus afinidades. También consideran como pertenecientes a Hemipogon: H. acerosus, $H$. abietoides, $H$. hemipogonoides y $H$. hatschbachii.

Tal afinidad en dicho grupo fue corroborada posteriormente por estudios filogenéticos basados en datos moleculares (Rapini et al., 2003; Rapini et al., 2007; Silva et al. 2012). En los dos primeros se utilizaron las regiones intrónicas trnL y el espaciador intergénico trnL-F del ADN plastidial. En el último se combinaron tanto marcadores plastidiales (trnT-F, trnS-G, trnH-psbA, trnD-T, rps 16, y matK), como nucleares (ITS). En todos ellos $H$. carassenssis y $H$. luteus forman un clado separado de $H$. acerosus, especie tipo del género. En la contribución de Silva et al. (2012), autores que analizaron el mayor número de especies de Hemipogon en estudios moleculares hasta el momento, H. carassenssis y H. luteus se presentan asociadas a otras especies de la Cadena del Espinazo de Minas Gerais (H. abietoides, $H$. hemipogonoides y $H$. hatschbachii), formando un clado mayor y bien sustentado ( $\mathrm{BS} \geq 95 \%$ ), denominado informalmente por los autores como clado "Hemipogon from Espinhaço Range".

Sin embargo, reunir a todos estos taxones 
en un solo género parece inadecuado debido a las diferencias morfológicas entre las especies del "Grupo luteus" sensu Rapini et al. (2001) y las demás. Por otro lado, este clado mayor se divide en dos ramas que se separan claramente en dos grupos, uno constituido por $H$. luteus y $H$. carassensis, otro por $H$. hemipogonoides, $H$. hatschbachii y $H$. abietoides, ambos con buena sustentación (BS $\geq 95 \%$ ), lo que corrobora los resultados morfológicos.

Con respecto al resto del género, ninguna conclusión puede ser inferida con los datos actuales, dado que todavía existen problemas dentro del grupo. H. acerosus, por ejemplo, se encuentra separado de las demás especies del género, formando un clado con Nephradenia acerosa Decne. fuera del "core group" de Metastelmatinae (Silva et al. 2012; LiedeSchumann \& Meve, 2013). Por otro lado, en los estudios de Liede-Schumann et al. (2005), Rapini et al. (2007), Silva et al. (2012) y, más recientemente, de Liede-Schumann \& Meve (2013), H. sprucei (especie tipo de Hemipogon subgen. Astephanopsis Malme) aparece a veces en un clado bien sustentado con $H$. andinus o con especies morfológicamente bien distintas como Blepharodon manicatum (Decne.) Fontella, Blepharodon bicuspidatum E. Fourn., Blepharodon pictum (Vahl) W. D. Stevens, Blepharodon grandiflorum Benth., Blepharodon glaucescens (Decne.) Fontella, Blepharodon mucronatum (Schltdl.) Decne., Ditassa angustifolia Decne. y Nephradenia filipes Malme.

Hemipogon sprucei, $H$. andinum y $N$. filipes tienen hábito erecto, más raro escandente, hojas opuestas, lineares o linear-lanceoladas, corola urceolada o anchamente campanulada y corona ausente o vestigial. Blepharodon manicatum, B. bicuspidatum, B. pictum, B. grandiflorum y $B$. glaucescens son arbustos volubles con hojas ovadas a oblongas, corola rotácea y corona bien desarrollada. D. angustifolia, también voluble, con hojas angostamente oblongas o estrechamente obovadas, posee corola rotácea y corona doble como las especies del género Ditassa R. Br.

Esta diversidad morfológica tan grande demuestra la necesidad de estudios más integrales en este grupo, sobre la base del examen de nuevas colecciones.

\section{Agradecimientos}

Al Conselho Nacional de Desenvolvimento Científico e Tecnológico (CNPq) de Brasil por la beca concedida al primer autor. A los curadores de los herbarios consultados de Brasil y del exterior. Al Dr. Ruy Valka Alves, Profesor de Botánica del Museu Nacional por las sugerencias. A los revisores por sus comentarios y sugerencias.

\section{Bibliografía}

DECAISNE, J. 1844. Asclepiadeae. En Candolle, A. L. P. P. de (ed.), Prodr. VIII, pp. 490-665. Treuttel \& Würtz, Paris.

ENDRESS, M., LIEDE-SCHUMANN, S. \& U. MEVE. 2014. An updated classification for Apocynaceae. Phytotaxa 159: 175-194.

FONTELLA PEREIRA, J. 1987. Estudos em Asclepiadaceae, II. Considerações sobre a ocorrência do gênero Astephanus R. Br. no Brasil. Bradea 4: 377-383, est. 1-4.

_ \& N. F. da S. MARQUETE. 1975. Estudos em Asclepiadaceae, VI. Uma nova espécie de Astephanus R. Br. Bradea 2: 9-12.

_ — \& E. de A. SCHWARZ. 1983. Estudos em Asclepiadaceae, XVIII. Bradea 4: 13-20.

_ \& R. J. PAIXÃO. 1996. Contribuição ao estudo das Asclepiadaceae Brasileiras, XXVII. Uma nova espécie de Hemipogon Decne. Bradea 6: 424-426.

FOURNIER, E. 1882. Sur les Asclépiadées américaines. Ann. Sci. Nat., Bot. $6^{\circ}$ sér., 14: 364-389.

. 1885. Asclepiadaceae. En Martius C. F. P., von Eichler A. G., Urban I (eds.) Flora Bras. 6: 27-331.

GOYDER, D. J. 2004. An amplified concept of Philibertia Kunth (Apocynaceae: Asclepiadoideae), with a synopsis of the genus. Kew Bull. 59: 415-451.

HOENE, F. C. 1951. Asclepiadaceae. En Índice bibliográfico e numérico das plantas colhidas pela comissão Rondon, p. 332. Secretaria da Agricultura do Estado de São Paulo.

LIEDE, S. 1994. Myth and reality of the subtribe Astephaninae (Decne.) Schumann (Asclepiadaceae). Bot. J. Linn. Soc. 114: 81-98.

- S. 1997. Subtribes and genera of the tribe Asclepiadeae (Apocynaceae - Asclepiadoideae) a synopsis. Taxon 46: 233-247.

- \& A. TAÜBER. 2002. Circumscription of the genus Cynanchum (Apocynaceae-Asclepiadoideae). Syst. Bot. 27: 789-801.

LIEDE-SCHUMANN, S., A. RAPINI, D. J. GOYDER $\&$ M. W. CHASE. 2005. Phylogenetics of the new world Subtribes of Asclepiadeae (Apocynaceae- 
Asclepiadoideae): Metastelmatinae, Oxypetalinae and Gonolobinae. Syst. Bot. 30: 184-195.

LIEDE-SCHUMANN, S. \& U. MEVE. 2013. The Orthosiinae revisited (Apocynaceae, Asclepiadoideae, Asclepiadeae). Ann. Missouri Bot. Gard. 99: 44-81.

MALME, G. O. A. 1900. Die Asclepiadaceen des Regnell'schen Herbars. Kongl. Svenska Vetensk. Acad. Handl. 34: 1-102, fig. t. 1-8.

G. O. A. 1927. Asclepiadaceae matogrossenses. Ark. Bot. 21A: 1-27.

RAPINI,A., R. deMELLO-SILVAR\& M.L.KAWASAKI. 2001. Asclepiadoideae: (Apocynaceae) da Cadeia do Espinhaço de Minas Gerais, Brasil. Bol. Bot. Univ. São Paulo 19: 55-169.

RAPINI, A., M. W. CHASE, D. J. GOYDER, \& J. GRIRIFFITHS. 2003. Asclepiadeae classification: evaluating the phylogenetic relationships of New World Asclepiadoideae (Apocynaceae). Taxon 52: 33-50.

RAPINI, A., CHASE, M. W. \& T. U. P. KONNO. 2006. Phylogenetics of South American Asclepiadeae (Apocynaceae). Taxon 55: 119-124.
RAPINI. A., C. van den BERG \& S. LIEDESCHUMANN. 2007. Diversification of Asclepiadoideae (Apocynaceae) in the New World. Ann. Missouri Bot. Gard. 94: 407-422.

RUSBY, H. H. 1920. Hemipogon. En Descriptions of Three Hundred New Species of South American Plants, pp. 92-93. Published by the author, New York.

SCHUMANN, K. 1895. Asclepiadaceae. En Engler, H.G.A. \& K. A. E. Prantl (eds.). Nat. Pflanzenfam. IV(2): 189-306. Leipzig (Wilhelm Engelmann). . 1898. Asclepiadaceae. En Urban, I. Plantae novae americanae imprimis Glaziovianae, II. Bot. Jahrb. Syst. 25: 19-23.

SILVA, U. C. S., A. RAPINI, S. LIEDE-SCHUMANN, P. L. RIBEIRO, \& C. VAN DEN BERG. 2012. Taxonomic considerations on Metastelmatinae (Apocynaceae) based on plastid and nuclear DNA. Syst. Bot. 37: 795-806.

THIERS, B. 2013. Index Herbariorum: A global directory of public herbaria and associated staff. New York Botanical Garden's Virtual Herbarium. http:// sweetgum.nybg.org/ih/ (último acceso 2 mar. 2013).

Original recibido el 2 de diciembre de 2013; aceptado el 1 de abril de 2014. 